\title{
Asymptotic behavior of BV solutions to the equations of nonlinear viscoelasticity
}

\author{
Constantine M. Dafermos
}

For Marshall Slemrod on the occasion of his 70th birthday

It is shown that the total variation of admissible BV solutions to the Cauchy problem for a system modeling the equations of motion of one-dimensional nonlinear viscoelastic media with fading memory decays to zero as $t \rightarrow \infty$.

\section{Introduction}

The equations of motion of one-dimensional viscoelastic media with fading memory are modeled by simple systems in the form

$$
\left\{\begin{array}{l}
\partial_{t} u-\partial_{x} v=0 \\
\partial_{t} v-\partial_{x} f(u)-\int_{-\infty}^{t} a^{\prime}(t-\tau) \partial_{x} u d \tau=0
\end{array}\right.
$$

where $u$ denotes the strain and $v$ stands for velocity. The function $f$, encoding the instantaneous elastic response of the medium, is strictly increasing, normalized by $f^{\prime}(0)=1$, and nonlinear.

Under suitable assumptions on the relaxation kernel $a,(1.1)$ exhibits the typical behavior of hyperbolic systems of balance laws with relaxation: Smooth and small initial data generate globally defined smooth solutions to the Cauchy problem [6]. However, when the gradient of (even smooth) initial data gets large, waves break and shocks develop [1]. In that case one may hope, at best, for the existence of weak solutions, in the large.

As shown in [3], when the initial data have small total variation and decay rapidly to zero as $|x| \rightarrow \infty$, the Cauchy problem for (1.1) possesses a unique admissible BV solution, defined on the entire upper half-plane. The aim here is to demonstrate that the total variation of this solution decays to zero, as $t \rightarrow \infty$.

At the outset, in order to simplify the analysis, we make the assumption that the relaxation kernel is in the form

$$
a(t)=\frac{1}{\mu} e^{-\mu t} .
$$


The parameter $\mu$ is fixed bigger than 1 , in order to secure that the relaxed response of the medium is elastic.

For the above choice of relaxation kernel, and upon introducing the internal variable $w$,

$$
w(x, t)=\int_{-\infty}^{t} e^{-\mu(t-\tau)} u(x, \tau) d \tau
$$

(1.1) reduces to the strictly hyperbolic system

$$
\left\{\begin{array}{l}
\partial_{t} u-\partial_{x} v=0 \\
\partial_{t} v-\partial_{x} f(u)+\partial_{x} w=0 \\
\partial_{t} w-u+\mu w=0
\end{array}\right.
$$

We prescribe initial data

$$
u(x, 0)=u_{0}(x), v(x, 0)=v_{0}(x), w(x, 0)=w_{0}(x),-\infty<x<\infty,
$$

where $\left(u_{0}, v_{0}, w_{0}\right)$ have bounded variation,

$$
\operatorname{TV} u_{0}(\cdot)+\operatorname{TV} v_{0}(\cdot)+\operatorname{TV} w_{0}(\cdot)=\delta
$$

and decay to zero, as $|x| \rightarrow \infty$, so that

$$
\int_{-\infty}^{\infty}(1+|x|)^{2 s}\left[u_{0}^{2}(x)+v_{0}^{2}(x)+w_{0}^{2}(x)\right] d x=\sigma^{2},
$$

for some $s>1$. Furthermore, in order to eliminate trivial rigid motions,

$$
\int_{-\infty}^{\infty} u_{0}(x) d x=\int_{-\infty}^{\infty} v_{0}(x) d x=0 .
$$

The principal result is

Theorem 1.1 There are positive numbers $\delta_{0}$ and $\sigma_{0}$ such that when the initial data $\left(u_{0}, v_{0}, w_{0}\right)$ satisfy (1.6), for $\delta<\delta_{0}$, (1.7), for $\sigma<\sigma_{0}$, and (1.8), then the Cauchy problem (1.4), (1.5) possesses a unique admissible $\mathrm{BV}$ solution $(u, v, w)$, defined on $(-\infty, \infty) \times[0, \infty)$. Furthermore,

$$
\operatorname{TV} u(\cdot, t)+\operatorname{TV} v(\cdot, t)+\operatorname{TV} w(\cdot, t) \rightarrow 0, \quad \text { as } t \rightarrow \infty
$$

The assertion on the existence of the solution has already been established in [3] and [4], so it will be assumed known. Furthermore, the total 
variation of the solution over $(-\infty, \infty)$ remains bounded and small, uniformly in time on $[0, \infty)$ :

$$
\operatorname{TV} u(\cdot, t)+\operatorname{TV} v(\cdot, t)+\operatorname{TV} w(\cdot, t) \leq a \delta+b \sigma, \quad 0 \leq t<\infty
$$

The goal here is to verify the statement (1.9). For that purpose, we shall draw freely from [2], [4] and [5].

\section{Energy estimates and decay of the $L^{1}$ norm}

Let $(u, v, w)$ be the admissible BV solution to the Cauchy problem (1.4), (1.5), with initial data $\left(u_{0}, v_{0}, w_{0}\right)$ satisfying (1.6), (1.7) and (1.8), which is defined on $(-\infty, \infty) \times[0, \infty)$ and takes values in a small neighborhood of the origin. To avoid proliferation of symbols, we shall employ throughout $c$ as a generic positive constant that may depend on $\mu$ and on bounds of $f$ and its derivatives on a small neighborhood of the origin, but is independent of $\delta$ and $\sigma$.

The system (1.4) is endowed with an entropy-entropy flux pair

$$
\eta=g(u)+\frac{1}{2} v^{2}+\frac{1}{2} \mu w^{2}-u w, \quad q=-v f(u)+v w,
$$

where

$$
g(u)=\int_{0}^{u} f(\omega) d \omega
$$

Since $f^{\prime}(0)=1$ and $\mu>1, \eta$ is convex on the range of the solution, which is admissible, and thus

$$
\partial_{t} \eta(u, v, w)+\partial_{x} q(u, v, w)+r(u, w) \leq 0,
$$

in the sense of distributions, where $r$ is the entropy production,

$$
r(u, w)=(u-\mu w)^{2} .
$$

Lemma 2.1 We have

$$
\int_{0}^{\infty} \int_{-\infty}^{\infty}\left[u^{2}(x, t)+v^{2}(x, t)+w^{2}(x, t)\right] d x d t \leq c \sigma^{2} .
$$


Proof. It is taken from [4]; see also [7]. We introduce the "potential" functions

$$
\phi(x, t)=\int_{-\infty}^{x} u(y, t) d y, \quad \psi(x, t)=\int_{-\infty}^{x} v(y, t) d y
$$

Note that, by virtue of (1.7) and (1.8),

$$
\int_{-\infty}^{\infty}\left[\phi^{2}(x, 0)+\psi^{2}(x, 0)\right] d x \leq \frac{\sigma^{2}}{2(s-1)(2 s-1)} .
$$

We now define the functions

$$
\begin{gathered}
H=\phi^{2}+\frac{1}{\mu} w^{2}+\frac{1}{\mu(\mu-1)}[\mu \psi-w]^{2}-\frac{1}{2 \mu^{2}} \phi v \\
Q=-2 \phi \psi+\frac{1}{2 \mu^{2}} \phi f(u)-\frac{1}{2 \mu^{2}} \phi w \\
R=2 w^{2}+\frac{1}{2 \mu^{2}} v^{2}-\frac{1}{2 \mu^{2}} u^{2}+\frac{1}{2 \mu^{2}} u w+\Theta,
\end{gathered}
$$

where

$$
\Theta=\left[-\frac{1}{2 \mu^{2}} u+\frac{2}{\mu-1} w-\frac{2 \mu}{\mu-1} \psi\right][f(u)-u]
$$

After a laborious but straightforward calculation,

$$
\partial_{t} H(v, w, \phi, \psi)+\partial_{x} Q(u, w, \phi, \psi)+R(u, v, w, \psi)=0
$$

Furthermore, it is easy to see that

$$
\begin{gathered}
\eta(u, v, w)+H(v, w, \phi, \psi) \geq 0 \\
r(v, w)+R(u, v, w, \psi) \geq c\left(|u|^{2}+|v|^{2}+|w|^{2}\right) .
\end{gathered}
$$

Therefore, integrating the sum of $(2.3)$ and $(2.12)$ over $(-\infty, \infty) \times[0, \infty)$, we arrive at (2.5). This completes the proof.

Lemma 2.2 We have

$$
\begin{aligned}
& \int_{-\infty}^{\infty}\left[u^{2}(x, t)+v^{2}(x, t)+w^{2}(x, t)\right] d x \leq \frac{c \sigma^{2}}{t}, \quad 0<t<\infty \\
& t \int_{-\infty}^{\infty}\left[u^{2}(x, t)+v^{2}(x, t)+w^{2}(x, t)\right] d x \rightarrow 0, \quad \text { as } t \rightarrow \infty .
\end{aligned}
$$


Proof. By virtue of (2.5),

$$
\int_{0}^{\infty} \int_{-\infty}^{\infty} \eta(u(x, t), v(x, t), w(x, t)) d x d t \leq c \sigma^{2} .
$$

Moreover, by (2.3), $t \mapsto \int_{-\infty}^{\infty} \eta(u(x, t), v(x, t), w(x, t)) d x$ is nonincreasing and hence

$$
\int_{-\infty}^{\infty} \eta(u(x, t), v(x, t), w(x, t)) d x \leq \frac{c \sigma^{2}}{t}, \quad 0<t<\infty,
$$

whence (2.15) follows.

Fix any $\varepsilon>0$. On account of (2.17), there is $\tau>0$ such that

$$
\begin{gathered}
\tau \int_{-\infty}^{\infty} \eta(u(x, \tau), v(x, \tau), w(x, \tau)) d x<\frac{\varepsilon}{2} \\
\int_{\tau}^{\infty} \int_{-\infty}^{\infty} \eta(u(x, t), v(x, t), w(x, t)) d x d t<\frac{\varepsilon}{2} .
\end{gathered}
$$

By (2.3) and (2.4),

$$
\partial_{t}[t \eta(u, v, w)]+\partial_{x}[t q(u, v, w)] \leq \eta(u, v, w) .
$$

For any $t>\tau$, integrating (2.21) over $(-\infty, \infty) \times(\tau, t)$ and using (2.19), (2.20) we deduce

$$
t \int_{-\infty}^{\infty} \eta(u(x, t), v(x, t), w(x, t)) d x<\varepsilon, \quad \tau<t<\infty,
$$

which implies (2.16). The proof is complete.

\section{Lemma 2.3 Let}

$$
I(t)=\int_{-\infty}^{\infty}[|u(x, t)|+|v(x, t)|+|w(x, t)|] d x, \quad 0 \leq t<\infty .
$$

Then

$$
\begin{aligned}
& I(t) \leq c \sigma, \quad 0<t<\infty, \\
& I(t) \rightarrow 0, \quad \text { as } \quad t \rightarrow \infty .
\end{aligned}
$$

Proof. We fix $\lambda$ large enough for

$$
|q(u, v, w)| \leq \lambda \eta(u, v, w)
$$


for all $(u, v, w)$ in the range of the solution. We also fix any $t>0$ and for $m=0,1,2, \ldots$ we integrate $(2.3)$ over the set $\{(x, \tau): 0 \leq \tau \leq t$, $\lambda(t+\tau)+m \leq x<\infty\}$. Using (2.4) and (2.26),

$$
\int_{2 \lambda t+m}^{\infty} \eta(u(x, t), v(x, t), w(x, t)) d x \leq \int_{\lambda t+m}^{\infty} \eta\left(u_{0}(x), v_{0}(x), w_{0}(x)\right) d x
$$

On account of (1.7) this yields

$$
\int_{2 \lambda t+m}^{\infty}\left[u^{2}(x, t)+v^{2}(x, t)+w^{2}(x, t)\right] d x \leq c(1+\lambda t+m)^{-2 s} \sigma^{2} .
$$

Therefore, by Schwarz's inequality,

$$
\int_{2 \lambda t+m}^{2 \lambda t+m+1}[|u(x, t)|+|v(x, t)|+|w(x, t)|] d x \leq c \sigma(1+\lambda t+m)^{-s} .
$$

Since

$(2.30)$

$\sum_{m=0}^{\infty}(1+\lambda t+m)^{-s} \leq(1+\lambda t)^{-s}+\int_{0}^{\infty}(1+\lambda t+\xi)^{-s} d \xi \leq \frac{s}{s-1}(1+\lambda t)^{1-s}$,

(2.29) implies

$$
\int_{2 \lambda t}^{\infty}[|u(x, t)|+|v(x, t)|+|w(x, t)|] d x \leq c \sigma(1+\lambda t)^{1-s} .
$$

A similar argument yields

$$
\int_{-\infty}^{-2 \lambda t}[|u(x, t)|+|v(x, t)|+|w(x, t)|] d x \leq c \sigma(1+\lambda t)^{1-s} .
$$

At the same time, by Schwarz's inequality,

$$
\begin{aligned}
& \int_{-2 \lambda t}^{2 \lambda t}[|u(x, t)|+|v(x, t)|+|w(x, t)|] d x \\
& \quad \leq\left\{12 \lambda t \int_{-\infty}^{\infty}\left[u^{2}(x, t)+v^{2}(x, t)+w^{2}(x, t)\right] d x\right\}^{\frac{1}{2}} .
\end{aligned}
$$

Combining (2.31), (2.32), (2.33) and (2.15), we arrive at (2.24). Furthermore, (2.31), (2.32), (2.33) and (2.16), yield (2.25). The proof is complete. 


\section{Redistribution of damping}

Our system, in its present form (1.4), does not lend itself to establishing the decay of the total variation of solutions, because the first two equations appear damping-free while the source seems to induce damping only to the third equation. As explained in [2], one may escape this predicament by reformulating the system through a state vector transformation in such a manner that the damping is redistributed more equitably among the equations. For the case of (1.4), it is natural to pass from $(u, v, w)$ to the state vector $(u, z, \omega)$, where

$$
z=v+\frac{1}{2} \phi, \quad \omega=w-\psi,
$$

with $\phi$ and $\psi$ defined by (2.6). In terms of the new state vector, (1.4) takes the form

$$
\left\{\begin{array}{l}
\partial_{t} u-\partial_{x} z+\frac{1}{2} u=0 \\
\partial_{t} z-\partial_{x} f(u)+\partial_{x} \omega+\frac{1}{2} z=\frac{1}{4} \phi \\
\partial_{t} \omega+f(u)-u+(\mu-1) \omega=-(\mu-1) \psi
\end{array}\right.
$$

The initial conditions read

$u(x, 0)=u_{0}(x), z(x, 0)=z_{0}(x)=v_{0}(x)+\frac{1}{2} \phi(x, 0), \omega(x, 0)=w_{0}(x)-\psi(x, 0)$.

There is now a fair distribution of damping among the equations of (3.2) and thus, following [2], one may estimate the variation of the solution by combining the random choice method with operator splitting. In the process, $\phi$ and $\psi$ on the right-hand side of (3.2) are treated as known functions, whose contributions to the variation of $(u, z, \omega)$ is estimated through their own variation. By virtue of (2.6) and (2.23),

$$
\operatorname{TV} \phi(\cdot, t)+\operatorname{TV} \psi(\cdot, t) \leq I(t), \quad 0 \leq t<\infty .
$$

A detailed exposition of the estimation is found in $[2,5]$, so it will suffice to record here the conclusion:

$$
\begin{aligned}
& \operatorname{TV} u(\cdot, t)+\operatorname{TV} z(\cdot, t)+\operatorname{TV} \omega(\cdot, t) \\
& \quad \leq c e^{-\nu t}\left[\operatorname{TV} u_{0}(\cdot)+\operatorname{TV} z_{0}(\cdot)+\operatorname{TV} \omega_{0}(\cdot)\right]+c \int_{0}^{t} e^{-\nu(t-\xi)} I(\xi) d \xi
\end{aligned}
$$


for some $\nu>0$. In terms of the original state vector:

$$
\begin{aligned}
& \operatorname{TV} u(\cdot, t)+\operatorname{TV} v(\cdot, t)+\operatorname{TV} w(\cdot, t) \\
& \leq c e^{-\nu t}\left[\operatorname{TV} u_{0}(\cdot)+\operatorname{TV} v_{0}(\cdot)+\operatorname{TV} w_{0}(\cdot)\right] \\
& \quad+c I(t)+c \int_{0}^{t} e^{-\nu(t-\xi)} I(\xi) d \xi .
\end{aligned}
$$

Thus, upon using (1.6) and (2.24),

$$
\operatorname{TV} u(\cdot, t)+\operatorname{TV} v(\cdot, t)+\operatorname{TV} w(\cdot, t) \leq c \delta e^{-\nu t}+c \sigma .
$$

We finally write the analog of (3.6) for a pair of times $0 \leq \tau<t<\infty$ :

$$
\begin{aligned}
& \operatorname{TV} u(\cdot, t)+\operatorname{TV} v(\cdot, t)+\operatorname{TV} w(\cdot, t) \\
& \leq c e^{-\nu(t-\tau)}[\operatorname{TV} u(\cdot, \tau)+\operatorname{TV} v(\cdot, \tau)+\operatorname{TV} w(\cdot, \tau)] \\
& \quad+c I(t)+c \int_{\tau}^{t} e^{-\nu(t-\xi)} I(\xi) d \xi
\end{aligned}
$$

On account of (3.7) and (2.25), given any $\varepsilon>0$, one can find $0 \leq \tau<\tilde{t}<\infty$, depending on $\delta, \sigma$ and $\nu$, such that the right-hand side of (3.8) becomes less than $\varepsilon$, for all $t \geq \tilde{t}$. We thus arrive at (1.9), and the proof of Theorem 1.1 is now complete.

\section{References}

[1] C. M. Dafermos, Development of singularities in the motion of materials with fading memory. Arch. Rational Mech. Anal. 91 (1986), 193-205. MR0806001

[2] C. M. Dafermos, Hyperbolic systems of balance laws with weak dissipation. J. Hyperbolic Differ. Equ. 3 (2006), 507-527. MR2238740

[3] C. M. Dafermos, Redistribution of damping in viscoelasticity. Communications in PDE 38 (2013), 1274-1286. MR3169746

[4] C. M. Dafermos, BV solutions for hyperbolic systems of balance laws with relaxation. J. Differential Equations 255 (2013), 2521-2533. MR3082473

[5] C. M. Dafermos, Asymptotic behavior of BV solutions to hyperbolic systems of balance laws with relaxation. J. Hyperbolic Differ. Equ. (to appear). 
[6] W. J. Hrusa, J. A. Nohel, The Cauchy problem in one-dimensional nonlinear viscoelasticity. J. Differential Equations 59 (1985), 388-412. MR0807854

[7] T. Ruggeri, D. Serre, Stability of constant equilibrium state for dissipative balance laws systems with a convex entropy. Quart. Appl. Math. 62 (2004), 163-179. MR2032577

Constantine M. Dafermos

Division of Applied Mathematics

Brown UnIVERSITY

Providence, Rhode Island 02912

USA

E-mail address: constantine_dafermos@brown.edu

Received September 24, 2013 